'Servicio de Neurología, Hospital del Salvador. Santiago, Chile.

${ }^{2}$ Departamento de Ciencias Neurológicas Oriente, Universidad de Chile. Santiago, Chile. ${ }^{3}$ Programa adultos, Centro de Salud Familiar Dr. Aníbal Ariztía. Santiago, Chile.

${ }^{4}$ Unidad de Metodología de la Investigación, Fundación Arturo López Pérez. Santiago, Chile. 5Unidad de Neuropsicología, Especialidades médicas L y $S$.

${ }^{a}$ Residente de Neurología.

bSociólogo, Magíster en Epidemiología. 'Psicóloga. dPhD.

Los autores no declaran conflicto de intereses.

Trabajo no recibió financiamiento.

Recibido el 10 de enero de 2017 , aceptado el 27 de julio de 2017.

Correspondencia a:

Dr. Daniel Jiménez Departamento de Ciencias Neurológicas, Facultad de Medicina, Universidad de Chile. José Miguel Infante 553, Providencia, Santiago, Chile. danieljimenez@med.uchile.cl

\section{Evaluación del minimental abreviado de la evaluación funcional del adulto mayor (EFAM) como screening para la detección de demencia en la atención primaria}

\author{
DANIEL JIMÉNEZ ${ }^{1,2}$, MANUEL LAVADOS ${ }^{2}$, \\ PAULA ROJAS ${ }^{3}$, CLAUDIO HENRÍQUEZ ${ }^{2, a}$, \\ FERNANDO SILVA ${ }^{4, \mathrm{~b}}$, MARTA GUILLÓN ${ }^{5, \mathrm{c}, \mathrm{d}}$
}

\section{Performance of an abbreviated mini mental examination to detect dementia in older people}

Background: The usefulness of the abbreviated Mini-Mental State Examination included in the Chilean Functional assessment of elderly people (MMSE-EFAM) to detect Dementia has not been determined. Aim: To assess the performance of the MMSE-EFAM to detect dementia. Material and Methods: We studied a non-probabilistic sample of subjects older than 65 years who had been assessed by the MMSE-EFAM in a Chilean primary care center during a period of 6 months. Patients underwent clinical evaluation by a neurologist blinded to MMSE-EFAM score, to establish the diagnosis of dementia using DSM-IV-TR criteria. Besides, the full Mini-Mental State Examination (MMSE) was applied. Results: The clinical diagnosis of Dementia was established in 13 of the 54 peoples evaluated. MMSE-EFAM had a sensitivity of $30.8 \%$ (95\% confidence intervals (CI); 9-61.4) and a specificity of 90.2\% (95\% CI; 76.9\%-97.3\%), while MMSE had a sensitivity of $84.6 \%$ (95\% CI; 54.6-98.1) and a specificity of 58.5\% (95\% CI; 42.1-73.7). In a receiver operating characteristic (ROC) curve analysis, the areas under the curve (AUC) were 0.77 (95\% CI; 0.61-0.93) and 0.82 (95\% CI; 0.70-0.95) for MMSE-EFAM and MMSE, respectively. Socio-demographic variables did not influence test performance in both cases. Conclusions: MMSE-EFAM has a low sensitivity to detect patients with Dementia and it is not an effective screening tool. These results are in agreement with the evidence and international guidelines that do not support the use of cognitive screening tools to detect dementia in the older general population.

(Rev Med Chile 2017; 145: 862-868)

Key words: Dementia; Mass screening; Neuropsychological Tests; Primary Health Care.
$\mathrm{E}$ 1 envejecimiento poblacional ha llevado consigo un aumento en la prevalencia de demencia. Alrededor de 24 millones de personas en el mundo tienen una demencia, y se estima que su prevalencia se doblará cada 20 años ${ }^{1}$. Un estudio epidemiológico reciente mostró que 13,9\% de los adultos de Estados Unidos de Norteamérica sobre 70 años padecen alguna forma de demencia, especialmente Alzheimer ${ }^{2}$. En Chile, se ha reportado una prevalencia de deterioro cognitivo de 5 a 9\% en mayores de 60-65 años, cifra que aumenta exponencialmente a partir de los 75 años $^{3,4}$. 
El diagnóstico suele establecerse en etapas tardías de la enfermedad ${ }^{5,6}$, documentándose una demora de 8 a 32 meses entre el comienzo de los síntomas y el diagnóstico de demencia ${ }^{7,8}$. Además, existe una clara asociación entre la severidad del deterioro cognitivo y la falla en su detección, hecho bien documentado en estudios que muestran que en las demencias leves los casos no diagnosticados pueden llegar a $95 \%{ }^{9,10}$.

Los centros de atención primaria suelen ser el primer contacto de los pacientes con el sistema de salud y por tanto juegan un rol crucial en la sospecha y derivación oportuna de los posibles casos. Entre las dificultades que enfrenta la atención primaria se cuentan la falta de entrenamiento clínico específico, el uso de instrumentos de diagnóstico inadecuados, la ausencia de guías clínicas y la variable disponibilidad de servicios clínicos especializados ${ }^{11}$. Una de las estrategias propuestas para mejorar la oportunidad del diagnóstico es el uso de test cognitivos como parte del control médico anual de la población mayor de 65 años. Sin embargo, no hay evidencia que demuestre el beneficio del uso de este tipo de cribado poblacional en la atención primaria en el diagnóstico oportuno de demencia ${ }^{12}$.

En Chile, el examen anual de medicina preventiva del adulto mayor (EMPAM) aplicado en los centros de atención primaria, incluye la aplicación de una versión modificada del MMSE de Folstein como parte del ítem de Diagnóstico funcional del adulto mayor (EFAM-Chile) ${ }^{13}$. Esta versión del MMSE, que en este estudio llamaremos MMSE-EFAM, fue originalmente concebido como un instrumento abreviado para ser utilizado en el proyecto "Salud, Bienestar y Envejecimiento en América Latina y el Caribe" y fue desarrollado a partir del análisis de los datos de MMSE en población chilena obtenidos en el estudio "Demencia asociada a la edad" de la Organización Mundial de la Salud, realizado en la ciudad de Concepción entre los años 1990 y 1992³. Dicho estudio utilizó un MMSE modificado en los ítems atención/cálculo y dibujo, con el fin de disminuir la influencia de la baja escolaridad de la población estudiada ${ }^{14}$.

El MMSE-EFAM consta de 19 puntos y se considera alterado si el resultado es menor a 13, puntaje que mostró la mejor correlación con el punto de corte validado para la versión modificada del MMSE en Chile en el análisis de curvas ROC ${ }^{3}$. Los pacientes en quienes se pesquisa este resultado son derivados al médico para una evaluación clínica ${ }^{15}$. Es decir, se utiliza como un test de cribado poblacional previo a la evaluación clínica y se considera alterado bajo 13 puntos independiente de la presencia de síntomas y/o de un impacto funcional significativo.

El objetivo del presente estudio es evaluar el desempeño y la utilidad de esta versión modificada y abreviada del MMSE de Folstein como instrumento de cribado cognitivo en la población general de adultos mayores para la detección oportuna de pacientes con demencia en la atención primaria.

\section{Material y Método}

Se seleccionó una muestra no probabilística, por conveniencia, de todos los adultos mayores de 65 años del Consultorio Aníbal Ariztía que hubieran sido evaluados con el EFAM en un período de 6 meses por una de las enfermeras del consultorio que habitualmente realiza esta evaluación, y que cumplieran con los siguientes requisitos: 1) el EFAM se realizó por completo; 2) el MMSE abreviado del EFAM fue aplicado en todos sus ítems y su puntaje total documentado adecuadamente; 3 ) el paciente fue ubicable vía telefónica o por visita domiciliaria; 4) contaba con un acompañante para asistir a la evaluación; este acompañante mantiene contacto regular con el paciente; 5) el paciente y su acompañante aceptaron participar previa firma del consentimiento informado. Fueron excluidos de este estudio aquellos pacientes con limitaciones físicas o sensoriales que impidieron la aplicación del MMSE-EFAM y de Folstein, y quienes después de una segunda citación no acudieron con un acompañante adecuado.

Los participantes fueron evaluados por un neurólogo que no tuvo acceso al puntaje obtenido por el sujeto en el MMSE-EFAM. La evaluación clínica incluyó una entrevista semi estructurada y un examen neurológico completo, con el propósito de obtener la información clínica que permitiera afirmar o descartar el diagnóstico clínico de demencia de acuerdo a los criterios del DSMIV-TR ${ }^{16}$. Además de la evaluación de dominios cognitivos, la entrevista incluyó la exploración de antecedentes médico-quirúrgicos y de síntomas depresivos, cuya presencia no excluyó necesariamente el diagnóstico del síndrome demencial dada la frecuente coexistencia de ambas condiciones. 
Adicionalmente se aplicó una versión traducida al castellano del MMSE de Folstein original sin modificaciones e idéntica a la propuesta por Giménez-Roldán ${ }^{17}$. El desempeño en esta prueba fue considerado en el análisis clínico y estadístico, pero el puntaje obtenido no fue necesario ni suficiente para establecer el diagnóstico de demencia.

El presente estudio contó con la aprobación del Comité Ético Científico Metropolitano Oriente.

\section{Análisis estadístico}

Se utilizó para todos los cálculos el programa STATA 12 (StataCorp, College Station, TX, USA) licencia individual de FSR.

Las variables fueron resumidas utilizando medidas de tendencia central de acuerdo a la naturaleza de las escalas de medición. Se reporta media aritmética y desviación estándar para la edad, y medianas para las variables de tipo no-paramétricas MMSE-EFAM y MMSE de Folstein. Las variables escolaridad y sexo fueron resumidas en porcentajes.

Para evaluar las diferencias de puntajes MMSEEFAM y MMSE de Folstein entre sujetos dementes y no dementes realizamos la prueba U de Mann Whitney considerando estadísticamente significativos los resultados donde el valor $\mathrm{p}$ fuese menor a 0,05 .

Para identificar puntos de corte óptimos en relación al diagnóstico clínico se realizó un análisis ROC (receiving operative characteristic) tanto para el instrumento MMSE-EFAM como para el MMSE de Folstein, reportándose tanto el área bajo la curva como también la sensibilidad y especificidad.

Se pesquisó posible confusión de factores como el sexo o la escolaridad en cuanto a la clasificación de los instrumentos MMSE-EFAM y MMSE de Folstein en contraste al diagnóstico clínico de demencia (en puntos de corte seleccionados). Se ejecutó un análisis a través de regresión logística evaluando la influencia de estos dos factores y se consideró como confusión un valor p asociado al Odds Ratio de sexo o escolaridad menor a 0,05.

Para comparar los valores del área bajo la curva de los instrumentos MMSE-EFAM y MMSE de Folstein, se realizó la prueba Bonferroni-adjusted significance probability, considerando estadísticamente significativos aquellos resultados donde el valor $\mathrm{p}$ fue menor a 0,05 .

\section{Resultados}

Cumplieron los criterios de inclusión 54 sujetos quienes aceptaron participar del estudio. El promedio de edad fue de 76,4 años ( $\pm 7,7$ años) y $35(64,8 \%)$ de los participantes fueron mujeres. En cuanto al nivel educacional, $38(70,4 \%)$ tenían una escolaridad de 4 o más años. En 13 (24\%) sujetos se estableció el diagnóstico clínico de demencia de acuerdo a los criterios del DSM-IV.TR. Como se presenta en la Tabla 1, las variables socio demográficas (sexo, escolaridad y edad) no mostraron diferencias según el diagnóstico clínico.

Las medianas de puntaje en el MMSE-EFAM $y$ en el MMSE de Folstein aplicado por nosotros fueron significativamente distintas de acuerdo al diagnóstico clínico (Tabla 1, Figura 1). La mediana para el MMSE-EFAM fue de 13 (Rango intercuartílico [RIC] 8-15) en el caso de los su-

Tabla 1. Variables demográficas y puntajes de MMSE-EFAM y MMSE de Folstein en relación al diagnóstico de demencia

\begin{tabular}{|c|c|c|c|c|}
\hline Variables & $\begin{array}{c}\text { Total } \\
n=54\end{array}$ & $\begin{array}{c}\text { Diagnóstice } \\
\text { Sí } \\
n=13(24 \%)\end{array}$ & $\begin{array}{c}\text { demencia } \\
\text { No } \\
n=41(76 \%)\end{array}$ & p value \\
\hline Edad, media (DE) & $76,4 \quad(7,7)$ & $77,5(10,3)$ & $76,1 \quad(6,8)$ & $0,554^{\dagger}$ \\
\hline Mujeres, n (\%) & $35 \quad(64,8)$ & $(64,8)$ & $(65,9)$ & $0,776^{*}$ \\
\hline Escolaridad $\geq 4$ años, n (\%) & $(70,4)$ & $(53,9)$ & $(75,6)$ & $0,134^{*}$ \\
\hline MMSE-EFAM, mediana (RIC) & $16,5(13-19)$ & $(8-15)$ & $(15-19)$ & $0,002^{\S}$ \\
\hline MMSE Folstein, mediana (RIC) & $(20-26)$ & $(12-22)$ & $(22-27)$ & $<0,001^{\S}$ \\
\hline
\end{tabular}

*Basado en test $\chi^{2}$. ${ }^{\dagger}$ Basado en test $t$ de Student. ${ }^{\circledR}$ Basado en test $U$ de Mann-Whitney. $\mathrm{DE}=$ desviación estándar. RIC $=$ Rango inter cuartil. 


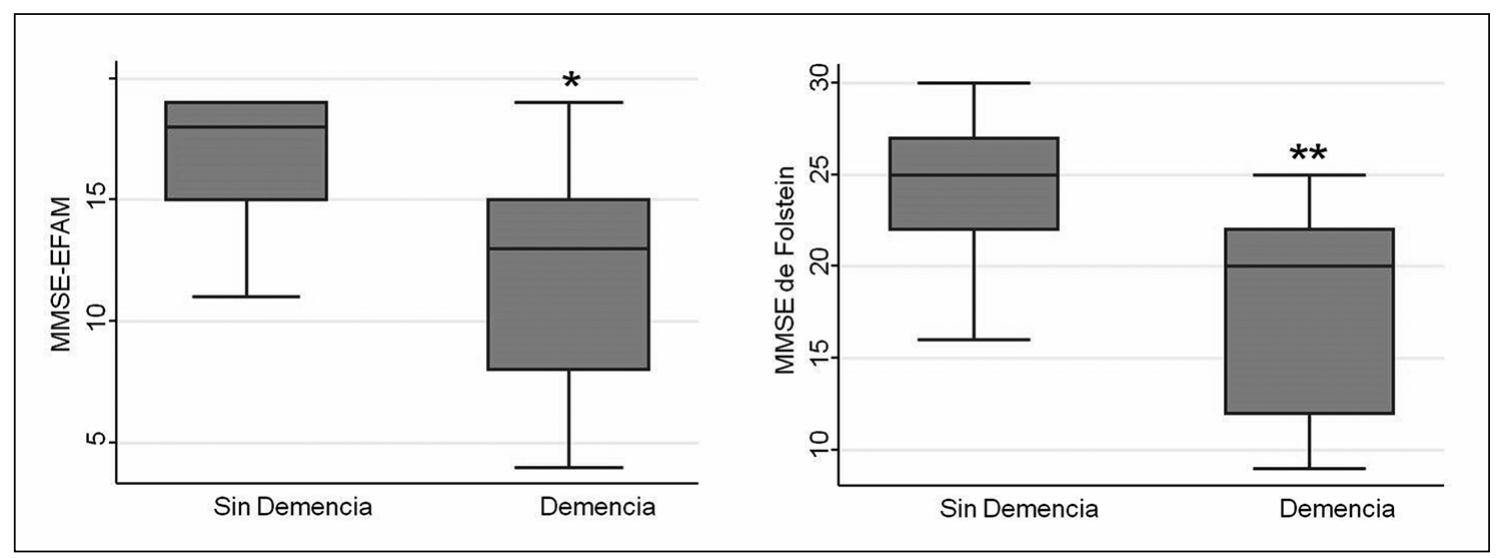

Figura 1. Puntajes de MMSE-EFAM y MMSE de Folstein en relación al diagnóstico clínico de demencia. ${ }^{*} p$ value $=0,002$; ${ }^{* *} p$ value $<0,001$ basado en test $U$ de Mann-Whitney.

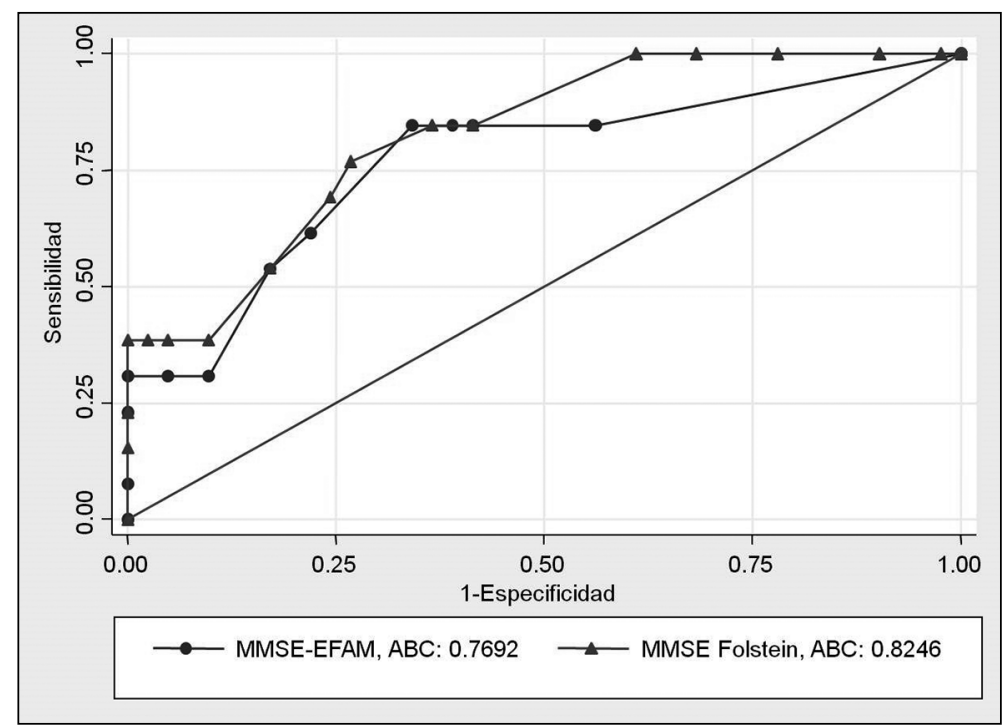

Figura 2. Curvas $R O C$ para $M M-$ SE-EFAM y MMSE de Folstein. ABC: Área bajo la curva. jetos con diagnóstico de demencia y de 18 (RIC 15-19) en quienes se consideraron sanos, siendo esta diferencia estadísticamente significativa ( $p$ value $<0,002$ ). Sin embargo, al examinar su rendimiento utilizando el punto de corte recomendado para la aplicación de este instrumento, el MMSE-EFAM alcanzó una sensibilidad de 30,8\% (95\% IC; 9-61,4) con una especificidad de 90,2\% (95\% IC; 76,9-97,3) para el diagnóstico clínico de demencia.

Los puntajes obtenidos en el MMSE de Folstein de Folstein aplicado por nosotros como parte de la evaluación clínica mostraron diferencia una estadísticamente significativa entre sujetos dementes y no dementes $(\mathrm{p}=<0,001)$, con medianas de 20 (RIC 12-22) y 25 (RIC 22-27) puntos respectivamente. Al examinar su rendimiento y utilizando el punto de corte habitual de 24/30 puntos, la sensibilidad fue de $84,6 \%$ (95\% IC; $54,6-98,1)$ y la especificidad de 58,5\% (95\% IC; 42,1-73,7).

En el análisis de las curvas ROC, el área bajo la curva para el MMSE-EFAM fue de 0,77 (95\% CI; 0,61-0,93), mientras que para el MMSE de Folstein fue de 0,82 (95\% CI; 0,70-0,95). Ambas 
curvas ROC se grafican en la Figura 2.

Las variables socio demográficas de la muestra, incluyendo sexo, edad y nivel educacional no influyen en el rendimiento de ambos instrumentos para la detección de los sujetos con demencia.

\section{Discusión}

La efectividad del uso del MMSE-EFAM en la atención primaria como herramienta de cribado poblacional para detectar demencia no había sido testeada. Nuestros resultados muestran que el rendimiento de MMSE-EFAM es insuficiente para ser utilizado como método de detección en la población general de adultos mayores. Aunque los puntajes obtenidos por los sujetos dementes y no dementes fueron significativamente distintos, la prueba mostró una baja sensibilidad y bajo poder discriminativo. El MMSE de Folstein aplicado por nosotros mostró una mejor sensibilidad contra el diagnóstico clínico de demencia, pero un poder de discriminación también insuficiente para ser utilizado como herramienta de screening.

Nuestros resultados permiten concluir que una versión del MMSE modificada y abreviada como la incluida en el EFAM no es una estrategia adecuada para identificar pacientes con demencia en la atención primaria. La distribución de los puntajes de MMSE-EFAM y MMSE de Folstein en sujetos con diagnóstico de demencia revela un grupo de sujetos con demencia catalogados como normales por los test (Figura 2). Una mayor escolaridad es una posible explicación para un mejor rendimiento en los test pese a tener historia clínica de deterioro de memoria respecto a su estado basal. Además, pacientes con presentaciones no amnésicas pueden no ser identificados por estas pruebas. Por contrapartida, hay un grupo de sujetos cognitivamente normales que fueron considerados sospechosos de demencia por haber obtenido menos de 13 puntos en el MMSE-EFAM. Aunque este instrumento adaptó el MMSE de Folstein con el objetivo de ser aplicable en una población de baja escolaridad, todos los sujetos identificados erróneamente como dementes en nuestra muestra tenían 4 o menos años de estudios.

El uso rutinario de test cognitivos como estrategia de cribado poblacional para la detección de demencia en la atención primaria continúa siendo controversial y objeto de crítica por la falta de evidencia que demuestre su beneficio ${ }^{18-20}$. Si bien existen numerosos estudios adecuadamente conducidos para evaluar la utilidad de los test cognitivos en adultos mayores no seleccionados, sólo unos pocos instrumentos han sido estudiados en condiciones aplicables a la atención primaria. En el caso particular de los test abreviados como MMSE-EFAM, la mayoría de las validaciones tienen la limitación metodológica de ser esencialmente correlativos, es decir, consisten en comparaciones entre distintos test que no son evaluados contra el diagnóstico clínico de demencia ${ }^{18,21}$. En 2013, el US Preventive Services Task Force actualizó sus recomendaciones para el screening de deterioro cognitivo en adultos mayores, basado en una extensa revisión sistemática de la literatura. Al igual que en su edición previa, el Task Force concluyó que no hay evidencia que demuestre el beneficio de este tipo de estrategias para el manejo y desenlace clínico de los pacientes con demencia ${ }^{12}$. Además, independiente del test a utilizar, el cribado de población no seleccionada no satisface los criterios establecidos por la OMS para un buen programa de screening ${ }^{18,22}$.

Pese a la falta de evidencia, varias guías y programas de detección de demencia recomiendan el cribado poblacional ${ }^{23}$. Uno de los principales argumentos para justificar su uso es el supuesto aumento en el número total de casos diagnosticados. Sin embargo, el seguimiento en el tiempo de este tipo de estrategias ha demostrado que aumenta el número de derivaciones a los centros secundarios a expensas de sujetos sanos, sin un impacto real en la oportunidad del diagnóstico de la demencia ${ }^{24}$. Una posible explicación para el mal rendimiento de este tipo de instrumentos es que el estándar de normalidad se define en relación al desempeño de una población de referencia y no al desempeño pre mórbido del sujeto. Además, a pesar de los intentos de adaptar las pruebas para poblaciones con altos niveles de analfabetismo, los test no pueden ser independientes del nivel educacional del individuo y de la población de referencia ${ }^{25}$.

En conclusión, la recomendación basada en la mejor calidad de evidencia disponible continúa siendo no utilizar test de cribado cognitivo en forma rutinaria ${ }^{12,20,26}$. Nuestros resultados corroboran el pobre desempeño de este tipo de instrumentos en los centros de Atención Primaria chilenos y constituyen una alerta que obliga a revisar las 
actuales estrategias para la detección de demencia. Desde una aproximación clínica, la sospecha de demencia debe basarse en el reconocimiento oportuno de un posible síndrome demencial cuando el paciente o su cuidador reportan los primeros síntomas de deterioro cognitivo al médico de la atención primaria ${ }^{11}$. La Academia Americana de Alzheimer ha propuesto un algoritmo cuyo primer paso consiste en una conversación entre el clínico, el paciente y algún familiar o informante, dirigida a identificar cambios recientes en la memoria $u$ otra función cognitiva y estimar su impacto en la funcionalidad del sujeto ${ }^{26}$. Estos elementos clínicos bastan para establecer la sospecha de demencia y derivar al centro de referencia respectivo para una evaluación por especialista, con el objetivo de confirmar el diagnóstico de demencia e identificar posibles causas reversibles o tratables.

\section{Referencias}

1. Ferri C, Prince M, Brayne C, Brodaty H. Global prevalence of dementia: a Delphi consensus study. Lancet 2006; 366 (9503): 2112-7.

2. Plassman BL, Langa KM, Fisher GG, Heeringa SG, Weir DR, Ofstedal MB, et al. Prevalence of dementia in the United States: The aging, demographics, and memory study. Neuroepidemiology 2007; 29 (1-2): 125-32.

3. Icaza MG, Albala C. Proyecto SABE. Minimental State Examinations (MMSE) del estudio de demencia en Chile: Análisis estadístico. Washington, D.C.; Organización Panamericana de la Salud; 1999 abril 24 p. Disponible en: http://www.bvsde.paho.org/texcom/ cd045364/049147.pdf

4. Ministerio de Desarrollo Social, Servicio Nacional del Adulto Mayor. Estudio Nacional de la Dependencia en las Personas Mayores. Santiago; Ministerio de Desarrollo Social, Chile; 2009120 p. Available from: http:// www.senama.cl/filesapp/Estudio_dependencia.pdf

5. Gifford DR, Cummings JL. Evaluating dementia screening tests Methodologic standards to rate their performance. Neurology 1999; 52: 224-7.

6. Van Hout H, Vernooij-Dassen M, Bakker K, Blom M, Grol R. General practitioners on dementia: Tasks, practices and obstacles. Patient Educ Couns 2000; 39 (2-3): 219-25.

7. Wilkinson D, Stave C, Keohane D, Vincenzino O. The role of general practitioners in the diagnosis and treatment of Alzheimer's disease: a multinational survey. J Int Med Res 2004; 32 (2): 149-59.
8. Bond J, Stave C, Sganga A, O’Connell B, Stanley RL. Inequalities in dementia care across Europe: key findings of the Facing Dementia Survey. Int J Clin Pract Suppl 2005; (146): 8-14.

9. Zunzunegui Pastor MV, del Ser T, Rodríguez Laso A, García Yebenes MJ, Domingo J, Otero Puime A. Non-detected dementia and use of the health services: implications for primary care. Aten Primaria 2003; 31 (9): 581-6.

10. Bermejo-Pareja F. Reflexiones sobre el cribado de enfermedades neurológicas. Neurología 2003; 18 Suppl 2: 29-38.

11. De Lepeleire J, Wind AW, Iliffe S, Moniz-Cook ED, Wilcock J, González VM, et al. The primary care diagnosis of dementia in Europe: an analysis using multidisciplinary, multinational expert groups. Aging Ment Health [Internet] 2008; 12 (5): 568-76.

12. Lin J, O'Connor E. Screening for cognitive impairment in older adults: a systematic review for the US Preventive Services Task Force. Ann Intern 2013; 159 (9): 601-12.

13. Ministerio de Salud, Subsecretaría de Salud Pública, División de Prevención y Control de Enfermedades. Manual de Aplicación del Examen de Medicina Preventiva del adulto mayor. Santiago; Ministerio de Salud, Chile; 2010. 16 p. Disponible en http://www.minsal.cl/portal/ url/item/ab1f81f43ef0c2a6e04001011e011907.pdf

14. Quiroga P, Albala C, Klaasen G. Validación de un test de tamizaje para el diagnóstico de demencia asociada a edad, en Chile. Rev Med Chile 2004; 467-78.

15. Donoso A, Fuentes P, Slachevsky A. Guías clínicas de trastornos cognitivos y demencias en el adulto mayor. Santiago; Ministerio de Salud, Chile; 2005. 68 p. Disponible en http://bibliotecaminsal-chile.bvsalud.org/lildbi/ docsonline/get.php?id=433

16. American Psychiatric Association Committee on Nomenclature and Statistics. Diagnostic and Statistical Manual of Mental Disorders (DSM-IV TR), 4th Edition. Washington, DC: American Psychiatric Association, 2000; 1995.

17. Giménez-Roldán S, Novillo MJ, Navarro E, Dobato JL, Giménez-Zuccarelli M. Mini-mental state examination: proposal of protocol to be used. Rev Neurol 1997; 25 (140): 576-83.

18. Brown J. The use and misuse of short cognitive tests in the diagnosis of dementia. J Neurol Neurosurg Psychiatry 2015; 86 (6): 680-5.

19. Couteur D, Doust J, Creasey H, Brayne C. Political drive to screen for pre-dementia : not evidence based and ignores the harms of diagnosis. BMJ Br Med J 2013; 5125 (September): 1-6.

20. Prince M, Comas-Herrera A, Knapp M, Guerchet M, 
Karagiannidou M. World Alzheimer Report 2016 Improving healthcare for people living with dementia 2016.

21. Velayudhan L, Ryu S-H, Raczek M, Philpot M, Lindesay J, Critchfield M, et al. Review of brief cognitive tests for patients with suspected dementia. Int Psychogeriatr 2014; 26 (8): 1247-62.

22. Wilson J, Jungner G. Principles and practice of screening for disease. Arch Intern Med 1969; 123 (3): 349.

23. NICE-SCIE D. The NICE-SCIE guideline on supporting people with dementia and their carers in health and social care. Br Psychol Soc R. 2006;

24. Menon R, Larner a J. Use of cognitive screening instru- ments in primary care: the impact of national dementia directives (NICE/SCIE, National Dementia Strategy). Fam Pract 2011; 28 (3): 272-6.

25. Woodford HJ, George J. Cognitive assessment in the elderly: a review of clinical methods. QJM 2007; 100 (8): 469-84.

26. Cordell CB, Borson S, Boustani M, Chodosh J, Reuben $\mathrm{D}$, Verghese J, et al. Alzheimer's Association recommendations for operationalizing the detection of cognitive impairment during the Medicare Annual Wellness Visit in a primary care setting. Alzheimers Dement. Elsevier Ltd 2013; 9 (2): 141-50. 